\section{Langzeittherapie der Schizophrenie mit Aripiprazol}

Claus Normann

Abtl. Psychiatrie und Psychotherapie, Universitätsklinikum Freiburg

psychoneuro 2004; 30 (12): 682-685

Da es sich bei der Schizophrenie um eine chronisch-rezidivierende Erkrankung mit hohem Rückfallrisiko handelt, sind sowohl Wirksamkeit als auch Verträglichkeit eines Antipsychotikums entscheidend für die Prognose eines an Schizophrenie Erkrankten. Aripiprazol ist ein vor kurzem zugelassenes Antipsychotikum mit einem neuartigen Wirkmechanismus. In klinischen Studien wurde die Wirksamkeit von Aripiprazol nicht nur bei akuten schizophrenen Exazerbationen, sondern auch in der Langzeittherapie schizophrener Patienten gezeigt. Die Substanz zeigte in Studien über sechs bis zwölf Monaten eine Plazebo überlegene Wirksamkeit und war gleichwertig mit Standard-Antipsychotika. Die Verträglichkeit war gut; insbesondere traten extrapyramidalmotorische Symptome, Gewichtszunahme und hormonelle Störungen nur relativ selten auf. Aripiprazol ist ein partieller Dopamin-Agonist und blockiert damit den Dopamin-Rezeptor bei einem pathologisch erhöhten Überangebot an Dopamin, während eine basale dopaminerge Neurotransmission erhalten bleibt.

$\mathrm{N}$ achdem in früheren Zeiten die „Dementia praecox“ noch die Dauerhospitalisierung tausender von Patienten in psychiatrischen Kliniken in der Größe ganzer Stadtteile notwendig gemacht hatte, hat sich die Langzeitprognose der Schizophrenie insbesondere nach der Einführung einer spezifischen und wirksamen Pharmakotherapie deutlich verbessert. Für die Betroffenen mündet die Diagnose einer schizophrenen Psychose jedoch auch heute noch in eine häufig lebenslange Problematik mit hohem Rückfallrisiko, die vollständige Wiederherstellung des prämorbiden Funktionsniveaus ist die Ausnahme. Eine hohe Zahl von Rückfällen führt nicht nur zu enormen gesellschaftlichen Kosten, sondern erhöht für den betroffenen Patienten auch das Risiko einer Chronifizierung und der Therapieresistenz. Eine hohe Zahl von Krankheitsepisoden beeinträchtigt die kognitiven Fähigkeiten und fördert möglicherweise die Entwicklung struktureller Hirnläsionen (6). Ohne suffizienten medikamentösen Schutz beträgt das Rückfallrisiko innerhalb eines Jahres etwa 50\%, nach zwei Jahren $80-90 \%(4,6)$. Eine langfristige Medikamenteneinnahme kann das Rückfallrisiko um mehr als $70 \%$ senken und das psychosoziale Funktionsniveau deutlich heben (8). Außerdem sind Rückfälle, die unter medikamentöser Prophylaxe auftreten, kürzer und weniger schwer (5). Einer der entscheidenden Faktoren zur Rückfallprophylaxe ist jedoch die langfristige Einnahme eines Antipsychotikums. In der Praxis ist dazu jedoch nur die Minderzahl der Patienten zu motivieren; Compliance ist eine der größten Limitationen der Schizophrenietherapie. Grundlegende Voraussetzung für die langfristige Einnahme eines Medikamentes ist ein günstiges Wirkungs-/Nebenwirkungsverhältnis.
Aripiprazol: Wirk-

mechanismus

Aripiprazol ist ein neues atypisches Antipsychotikum, dessen vermuteter Wirkmechanismus sich von denjenigen der bisher eingeführten Medikamente unterscheidet. Die Substanz ist ein partieller Antago-

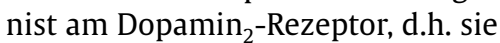
bindet an den Rezeptor und verhindert dessen Aktivierung durch endogenes Dopamin; im Gegensatz zu reinen $\mathrm{D}_{2}$-Antagonisten wie Haloperidol aktiviert Aripiprazol den Rezeptor jedoch zum Teil und hält eine partielle Aktivität aufrecht. In Experimenten an neuronalen Zellkulturen wird durch hohe Konzentrationen von Aripiprazol eine zirka 30\%ige Aktivierung des Dopamin ${ }_{2}$ Rezeptors erreicht $(1,12)$. Aufgrund dieser experimentellen Befunde ist zu erwarten, dass Aripiprazol eine dopaminerge Überstimulation verhindert, gleichzeitig jedoch eine basale Aktivität des Rezeptors aufrechterhält und dadurch die dopaminerge Neurotransmission stabilisiert.

Die Wirksamkeit von Aripiprazol zur Behandlung akuter schizophrener Episoden wurde bereits in mehreren Kurzzeitstudien bestätigt (2, 7 , 10, 14). Im Folgenden werden die Ergebnisse bisher vorliegender Langzeitstudien mit Aripiprazol zusammengefasst und bewertet.

\section{Ein-Jahres-Anwendung nach akuten Episoden: Vergleich mit Haloperidol}

Kasper et al. (11) berichten über die Ergebnisse zweier großer Studien, die gepoolt ausgewertet wurden. In diese Untersuchung wurden 1294 Patienten mit einer akuten Exazerbation einer vorbestehenden Schizophrenie eingeschlossen und für ein Jahr behandelt. Nachdem die Vormedikation mindestens fünf Tage ausgewaschen worden war, wurden die Studienteilnehmer doppelblind auf $30 \mathrm{mg}$ Aripiprazol oder $10 \mathrm{mg}$ Haloperidol randomisiert. Nach der ersten Woche konnten diese Dosen auf 20 mg Aripiprazol oder $7 \mathrm{mg}$ Haloperidol reduziert werden. Primäres Zielkriterium war die Zeit bis zum Verlust des Ansprechens auf die jeweilige Studien- 
medikation. Als Begleitmedikation waren Lorazepam und Anticholinergika zur Behandlung von extrapyramidal-motorischen Symptomen erlaubt.

43\% der Patienten in der Aripiprazol-Gruppe und 30\% der Studienteilnehmer unter Haloperidol beendeten die 52-wöchige Studienbehandlung (Abb. 1). Dieser signifikante Unterschied war im Wesentlichen auf eine höhere Abbruchrate aufgrund von Nebenwirkungen in der Haloperidol-Gruppe zurückzuführen. Zur insgesamt hohen $\mathrm{Ab}-$ bruchrate trug bei, dass etwa ein Viertel der Patienten aufgrund anderer Gründe aus der Studie ausschieden, z.B. Wegzug oder Nichterscheinen bei Visitenterminen. Eine ähnliche Abbruchrate wird bei schizophrenen Patienten beobachtet, die außerhalb klinischer Studien ambulant behandelt werden. Als Responder (mindestens 30\% Verbesserung im PANSS Total Score) wurden 52\% der Aripiprazol- und 44\% der Haloperidol-Patienten eingestuft; die Zeit bis zur erneuten Verschlechterung konnte unter Aripiprazol im Vergleich zu Haloperidol um 30\% verlängert werden. Die beiden Substanzen unterschieden sich nicht hinsichtlich ihrer Wirksamkeit auf die Positivsymptomatik; bezüglich der Negativsymptomatik und depressiver Symptome war Aripiprazol jedoch signifikant überlegen. So zeigte sich im PANSS negativ score eine signifikante Überlegenheit von Aripiprazol vs. Haloperidol (LOCF Analyse: $\mathrm{p}<0,05$ ). Die Verbesserung der depressiven Symptomatik gemessen anhand der Montgomery-Åsberg Depression Rating Scale (MADRS) war unter Aripiprazol ebenfalls signifikant größer als unter Haloperidol (LOCF: $p<0,05$ ).

Aripiprazol war insgesamt gut verträglich; es gab keinen Hinweis auf unerwünschte Wirkungen, die erst verzögert nach längerer Anwendung des Medikamentes auftraten. Mehr als die Hälfte aller Patienten unter Haloperidol entwickelten EPS und wurden mit Anticholinergika behandelt; in der Aripiprazol-Gruppe war dies bei weniger als einem Viertel der Fall. Es kam unter keinem der Medikamente zu einer Ge-

\section{Abb. 1 Verbleib in der 1-Jahres Studie}

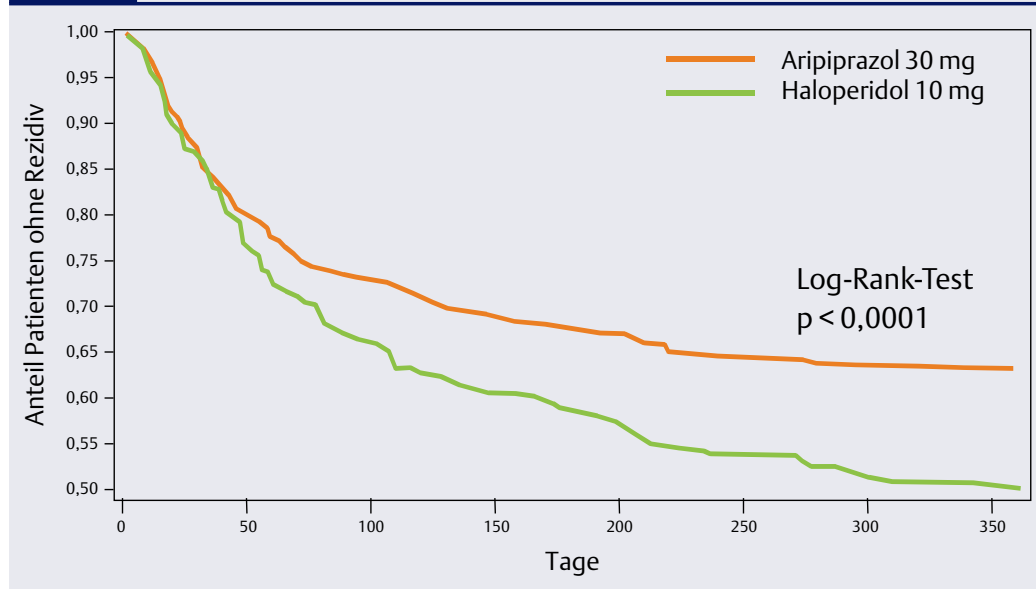

Verbleib in der 1-Jahres Studie nach Abbruch wegen Wirkungslosigkeit oder Nebenwirkungen. Das Risiko eines Behandlungsabbruchs war unter Aripiprazol um $31 \%$ geringer. wichtszunahme; unter Aripiprazol sanken die durchschnittlichen Prolaktinwerte um $8,1 \mathrm{ng} / \mathrm{ml}$, während sie unter Haloperidol um 34,2 ng/ml anstiegen. Unter der Haloperidoltherapie hatten 61\% der Patienten Prolaktinwerte über der Normgrenze, im Gegensatz zu lediglich 3,4\% der Patienten die mit Aripiprazol behandelt wurden. Zusammenfassend wies Aripiprazol in dieser Einjahresstudie in einem großen Patientenkollektiv eine dem Haloperidol vergleichbare Wirksamkeit in der Positivsymptomatik und eine überlegene Effektivität hinsichtlich der Negativsymptomatik und Depressivität auf; Aripiprazol war insgesamt deutlich besser verträglich als Haloperidol.

\section{Plazebo-kontrollierte Langzeitstudie bei chronischen Patienten}

In einer weiteren Langzeitstudie wurden teilremittierte, chronische Schizophreniepatienten für ein halbes Jahr plazebokontrolliert mit Aripiprazol behandelt (13). In die multizentrische Studie, durchgeführt in

\section{Abb. 2 Anteil der chronischen Patienten ohne Rückfall}

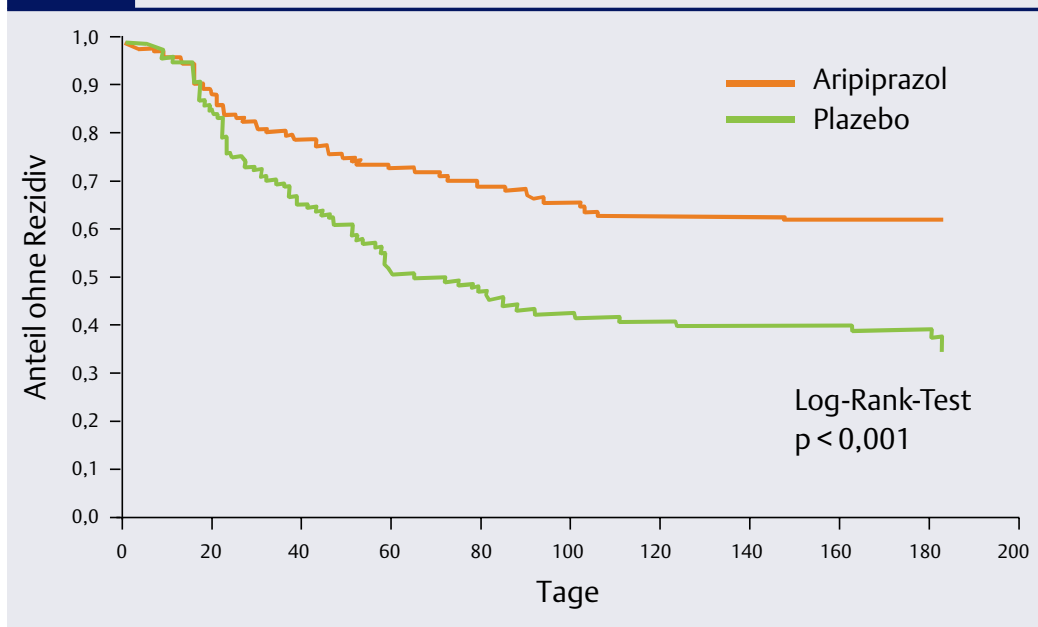

Anteil der chronischen Patienten, die unter Behandlung mit Aripiprazol oder Plazebo ohne Rückfall blieben. Die geschätzten Wahrscheinlichkeiten, am Ende der 26-wöchigen Behandlungsphase ohne Rückfall zu bleiben, betrug 63\% für Aripiprazol und $40 \%$ für Plazebo. Dieser Unterschied war signifikant. Das relative Risiko, einen Rückfall zu bekommen, war halbiert. 

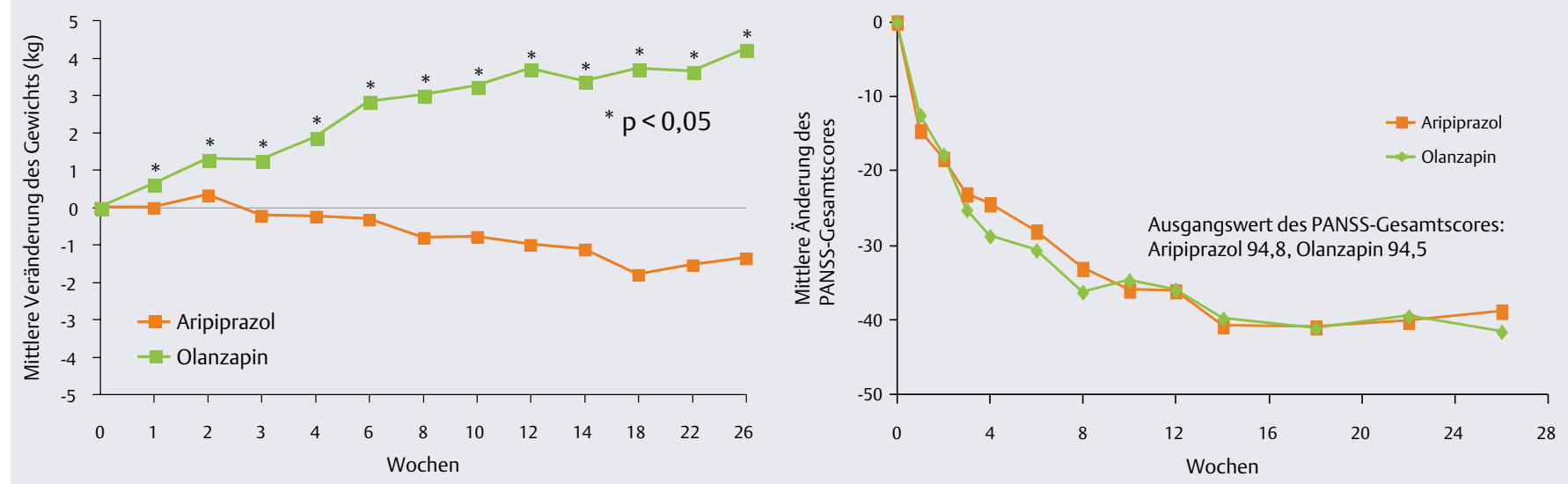

Veränderung des Körpergewichts und Abnahme des PANSS-Scores nach 26-wöchiger Therapie mit Aripiprazol und Olanzapin. Unter Olanzapin stieg das Körpergewicht um durchschnittlich 4 kg an, während es unter Aripiprazol zu einer nicht-signifikanten Gewichtsabnahme kam. Hinsichtlich ihrer Wirksamkeit unterschieden sich die Substanzen nicht.

den USA und Osteuropa, wurden 310 Patienten mit einem Durchschnittsalter von 42 Jahren eingeschlossen. Dabei handelte es sich um Patienten, die zum Zeitpunkt der Randomisierung mindestens drei Monate stabil gewesen, jedoch noch erheblich symptomatisch waren. Der durchschnittliche PANSS-Score lag bei 82 , dies entspricht einer moderaten Krankheitsschwere. Etwa die Hälfte der Patienten war auf Dauer in vollstationären Einrichtungen untergebracht, alle übrigen nahmen täglich teilstationäre oder ambulante psychiatrische Versorgungseinrichtungen in Anspruch. Weiterhin mussten alle Patienten vor Einschluss in die Studie eine Teilremission auf antipsychotische Medikation gezeigt haben. Primäres Zielkriterium der Studie war die Rückfallrate. Bereits eine Einstufung als „minimal schlechter“ im CGI-I oder eine Verschlechterung des PANSS um mehr als 20\% wurde als Rückfall gewertet, was im Vergleich zu anderen Studien eine sehr strikte Definition darstellt.

Nach der Randomisierung wurden die Patienten für 26 Wochen je zur Hälfte mit 15 mg/Tag Aripiprazol und mit Plazebo behandelt. Dies entspricht der empfohlenen Anfangs- und Erhaltungsdosis. Lorazepam und Anticholinergika waren als Begleitmedikation erlaubt. In der Plazebo-Gruppe kam es bei 57\% der Patienten zu einem Rückfall, in der Aripiprazol-Gruppe nur bei $34 \%$ (Abb. 2). Nach 26 Wochen verblie- ben noch 59\% der Aripiprazol-Patienten und 38\% der Plazebo-Patienten in der Studie. Da diese Studie bei „Stabilen“ Patienten mit Schizophrenie durchgeführt wurde, lagen die PANSS Werte deutlich niedriger als in der 1-Jahres Studie versus Haloperidol (ca. 95 vs. 82 PANSS Ausgangswerte). So zeigte sich im PANSS-Gesamtscore bei Patienten unter Aripiprazol eine Verbesserung um lediglich zwei Punkte, während es unter Plazebo zu einer Verschlechterung um 4,5 Punkte kam. In der PANSS-Positiv-Skala zeigte sich am Studienende auch in der Aripiprazol-Gruppe eine minimale Verschlechterung (Plazebo: +2,37; Aripiprazol: $+0,12$ ), die jedoch signifikant geringer als in der PlazeboGruppe war. In der PANSS-NegativSkala gab es keine signifikanten Unterschiede (Plazebo: -0,54; Aripiprazol:-1,40).

Unter Aripiprazol wurden etwas häufiger als unter Plazebo Tremor (Aripiprazol: 8,5\%; Plazebo: 1,3\%), Übelkeit (Aripiprazol: 5,2\%; Plazebo: 3,3\%), Erbrechen (Aripiprazol 5,9\%; Plazebo: 3,3\%) beobachtet. Vorbestehende EPS und Prolaktinerhöhungen besserten sich unter Aripiprazol stärker als unter Plazebo, es kam zu keinen Unterschieden hinsichtlich Blutfetten, Glukose, QT-Verlängerung oder Gewicht. Zusammenfassend kann aus dieser Studie geschlossen werden, dass es bei teilremittierten, noch erheblich symptomatischen Patienten nach Absetzen der Vormedikation unter Aripiprazol bei weniger Patienten zu einer klinischen Verschlechterung kommt als unter Plazebo. Bei dieser Studie handelt es sich mehr um eine Umstellungs- als um eine Rückfallprophylaxe-Studie, da die Patienten keine akute Exazerbation der Erkrankung aufwiesen und Absetzeffekte der vorangegangenen Medikation mit erheblicher Latenz auftreten können. In dieser schwerstkranken, zu einem hohen Prozentsatz dauerhospitalisierten und teilweise therapierefraktären Patientengruppe war Aripiprazol gegenüber Plazebo hinsichtlich seiner Wirksamkeit überlegen und gut verträglich.

Therapierefraktäre Patienten

Mit der Problematik therapierefraktärer Patienten beschäftigte sich eine weitere klinische Studie aus den USA (9). In diese wurden 300 Patienten eingeschlossen, die auf zwei oder mehr ausreichend dosierte und mehr als sechs Wochen angewandte Behandlungsversuche mit unterschiedlichen Neuroleptika nicht respondiert hatten. War zuvor Olanzapin erfolglos gewesen, wurden die Studienteilnehmer nochmals sechs Wochen offen mit Risperidon behandelt; war zuvor Risperidon ohne Effekt, wurde Olanzapin angesetzt. Darunter respondierten überraschenderweise nur $2 \%$ der Studienteilnehmer. Alle übrigen wurden danach auf Aripiprazol oder Perphenazin randomisiert. Perphenazin wurde hauptsächlich des- 
halb als Kontrollmedikation ausgewählt, da es als gut verträglich gilt und in den USA selten eingesetzt wird; dementsprechend gibt es nur wenige Patienten, die auf diese Substanz bereits refraktär sind. Etwa $75 \%$ der Patienten in beiden Gruppen beendeten die sechswöchige Behandlungsphase. In beiden Gruppen respondierten etwa ein Viertel der Patienten; in der Aripiprazol-Gruppe traten wiederum weniger EPS-Symptome und keine Hyperprolaktinämie auf. In beiden Behandlungsgruppen kam es zu einer geringen Gewichtsabnahme (Aripiprazol: -2,2 kg; Perphenazin: $-1,5 \mathrm{~kg}$ ). In dieser $\mathrm{Pa}-$ tientengruppe, deren Therapieresistenz sowohl gegenüber Risperidon als auch Olanzapin im Rahmen der Studie gezeigt wurde, konnten sowohl Aripiprazol als auch das hochpotente konventionelle Neuroleptikum Perphenazin noch bei einem signifikanten Prozentsatz der Patienten eine klinische Besserung herbeiführen; Aripiprazol wurde jedoch besser vertragen. Aus den Ergebnissen dieser Studie kann nicht zwangsläufig abgeleitet werden, dass Aripiprazol oder Perphenazin bei therapierefraktären Patienten gegenüber Olanzapin oder Risperidon überlegen sind, da bei längerer Dauer der medikamentösen Behandlung eine kumulative Zunahme der Responder zu erwarten ist und Aripiprazol/Perphenazin zeitlich nach Olanzapin/Risperidon eingesetzt wurden. Die Studie könnte jedoch auch so interpretiert werden, dass bei therapierefraktären Patienten von Substanzen mit alternativen Wirkmechanismen ein zusätzlicher Effekt erwartet werden könnte.

\section{Langfristige Wirkung auf Kognition und Gewicht}

Zwei weitere Langzeitstudien verwendeten Olanzapin als Vergleichssubstanz. In einer offenen Halbjahresstudie wurden 255 klinisch stabile Patienten auf $30 \mathrm{mg}$ Aripiprazol oder 10-15 mg Olanzapin umgestellt (3). Hauptzielkriterien dieser Studie waren eine Reihe von Tests zur neurokognitiven Funktion. Die PANSS-Gesamtwerte blieben in beiden Studienarmen im Verlauf der gesamten Untersuchung stabil und unterschieden sich nicht signifikant. Deutliche Unterschiede fanden sich beim sekundären Wortgedächtnis, also der Fähigkeit, verbale Informationen aufnehmen und länger als einige Minuten speichern zu können. Patienten unter Aripiprazol schnitten hier sowohl nach acht als auch nach 26 Wochen signifikant besser ab als die Olanzapin-Kontrollgruppe.

In eine weitere Halbjahresstudie wurden 314 Patienten mit akut exazerbierter Schizophrenie eingeschlossen und doppelblind mit durchschnittlich $25 \mathrm{mg}$ Aripiprazol oder 16 mg Olanzapin behandelt (15). Primäres Zielkriterium war hier die Veränderung des Körpergewichts. In dieser Studie zeigte sich, dass die Patienten im Verlauf des Studienzeitraumes unter Aripiprazol durchschnittlich leicht an Gewicht abnahmen $(-1,4 \mathrm{~kg})$, während mit Olanzapin behandelte Patienten im Durchschnitt um zirka vier $\mathrm{kg}$ zunahmen (Abb. 3, links). Diese Gewichtszunahme erreichte nach zirka zwölf Wochen ein Plateau. Auch in dieser Untersuchung unterschieden sich Aripiprazol und Olanzapin nicht hinsichtlich ihrer Wirksamkeit (Abb. 3, rechts).

\section{aazit}

In einer Reihe von Studien konnte für Aripiprazol eine gegenüber Plazebo überlegene und gegenüber typischen und atypischen Antipsychotika gleichwertige Wirksamkeit bei guter Verträglichkeit gezeigt werden. Insbesondere bei Umstellung von sedierenden Neuroleptika auf Aripiprazol scheint es bei manchen Patienten zu passageren Schlafstörungen zu kommen, so dass eine kurzfristige Komedikation mit einem Hypnotikum nötig werden kann. Von größerer Bedeutung für die Langzeittherapie ist jedoch die gute Verträglichkeit: Bewegungsstörungen, Prolaktinanstieg, EKG-Veränderungen und Gewichtszunahme treten nur selten unter Aripirazoltherapie auf. Die klinische Relevanz dieser Befunde aus klinischen Studien wird erst beurteilbar sein, wenn Aripiprazol in der klinischen Praxis breit angewendet wird.

\section{Literatur}

1. Burris KD, Moski TF, Xu C et al. Aripiprazole, a novel antipsychotic, is a high-affinity partial agonist at human dopamine $\mathrm{D}_{2}$ receptors. J Pharmacol Exp Ther 2002; 302: 381-389

2. Carson WH, Stock E, Saha AR et al. Meta-analysis of efficacy of aripiprazolee in the treatment of schizophrenia. Schizophrenia Res 2002; 53 (suppl 3): B96

3. Cornblatt B, Kern RS, Carson WH et al. Int ] Neuropychopharmacol 2002; 5 (suppl 1): $\mathrm{S} 185$

4. Crow TJ, McMillan JF, Johnson AL. The Norwhwick Park study of first episodes of schizophrenia, 2: a randomized controlled trial of prophylactic neuroleptic treatment. Br J Psychiatry 1986; 148:120-127

5. Davis JM, Kane JM, Marder SR et al. Dose response of prophylactic antipsychotics. J Clin Psychiatry 1993; 54: 24-30

6. DeQuardoJR. Pharmacologic treatment of first-episode schizophrenia: early intervention is key to outcome. J Clin Psychiatry 1998; 59 (suppl 19): 9-17

7. El Sayeh H, Morganti C. Aripiprazole for schizophrenia. Cochrane Database Syst Rev 2004; CD004578

8. Gilbert PL, Harris MJ, Mc Adams et al. Neuroleptic withdrawal in schizophrenic patients: a review of literature. Arch Gen Psychiatry 1995; 52:173-188

9. Kane J, Carson W, Kujawa $M$ et al. Aripiprazole vs. perphenazine in treatmentresistant schizophrenia. Poster präsentiert: Meeting of the American Psychiatric Association, 2003

10. Kane JM, Carson WH, Saha AR et al. Efficacy and Safety of aripiprazole and haloperidol versus placebo in patients with schizophrenia and schizoaffective disorder. J Clin Psychiatry 2002; 63: 763-771

11. Kasper S, Lerman M, McQuade RD et al. Efficacy and safety of aripiprazole vs. haloperidol for long-term maintenance treatment following acute relapse of schizophrenia. Int J Neuropsychopharmacol 2003; 6: 325-337

12. Kikuchi T, Tottori K, Uwahodo $\mathrm{Y}$ et al. OPC-14597, a new putative antipsychotic drug with both presynaptic and postsynaptic $\mathrm{D}_{2}$ receptor antagonist activity. J Pharmacol Exp Ther 1995; 274: 329-336

13. Pigott TA, Carson WH, Saha AR et al. For the Aripiprazole Study Group. Aripiprazole for the prevention of relapse in stabilized patients with chronic schizophrenie: a placebo-controlled 26-week study. J Clin Psychiatry 2003; 64: 1048-1056

14. Potkin SG, Saha AR, Kujawa MJ et al. Aripiprazole, an antipsychotic with a novel mechanism of action, and risperidone vs. placebo in patients with schizophrenia and schizoaffective disorder. Arch Gen Psychiatry 2003; 60: 681-690

15. Schmauss $M$, Ebrecht $M$, Kungel $M$ et al. Metabolische Parameter und Gewichtsveränderung unter Aripiprazol, Olanzapin und Placebo. Der Nervenarzt 2003; 74 (Suppl.2): 175

Korrespondenzadresse:

Dr. Claus Normann

Abtl. Psychiatrie und Psychotherapie

mit Poliklinik

Hauptstr. 5, 79104 Freiburg 